\title{
Clinical characteristics of patients with premature acute coronary syndrome and adverse cardiovascular events after PCI
}

\author{
XIAO-JUN SHI* ${ }^{*}$ MIAO-NAN LI ${ }^{*}$, LING XUAN, HUI LI, BIN CHEN, JUN ZHANG and HONG-JU WANG \\ Department of Cardiovascular Disease, The First Affiliated Hospital of Bengbu Medical College, \\ Anhui, Bengbu 233000, P.R. China
}

Received July 18, 2018; Accepted March 7, 2019

DOI: $10.3892 /$ etm.2019.7618

\begin{abstract}
Few studies have compared the clinical manifestations of patients with premature acute coronary syndrome (ACS) and late-onset ACS as well as the adverse cardiovascular events following percutaneous coronary intervention (PCI). To investigate the clinicopathological characteristics of patients with premature ACS and adverse cardiovascular events following PCI, a total of 726 patients with ACS undergoing PCI were divided into two groups: A premature ACS group and a late-onset ACS group. Following discharge, all patients were followed-up for an average of 23.5 \pm 5.3 months. Clinical characteristics, Gensini scores, vascular lesions and adverse cardiovascular events were compared between the two groups. There were no significant differences in smoking, diabetes, ACS composition ratio, baseline treatment of coronary heart disease, high-density lipoprotein level and C-reactive protein levels between the two groups. Sex and hypertriglyceridemia were determined to be independent risk factors of premature ACS, while age, hypertension and a high Gensini score were independent risk factors for adverse cardiovascular events in patients with ACS following PCI. Furthermore, the prevalence of premature ACS was significantly higher in females. Although serum levels of fasting blood glucose, total cholesterol, triglycerides and low-density lipoprotein were also significantly higher in
\end{abstract}

Correspondence to: Professor Hong-Ju Wang or Dr Ling Xuan, Department of Cardiovascular Disease, The First Affiliated Hospital of Bengbu Medical College, 287 Zhihuai Road, Anhui, Bengbu 233000, P.R. China

E-mail: hongjuwang1@sina.com

E-mail: 17893466@qq.com

*Contributed equally

Abbreviations: ACS, acute coronary syndrome; PCI, percutaneous coronary intervention; CAD, coronary artery disease; AF, atrial fibrillation; STEMI, ST-segment elevation myocardial infarction; NSTEMI, non-ST-segment elevation myocardial infarction

Key words: premature acute coronary syndrome, percutaneous coronary intervention, adverse cardiovascular events patients with premature ACS compared with patients with late-onset ACS, patients with premature ACS exhibited fewer vascular lesions compared with patients with late-onset ACS. Furthermore, the incidence of adverse cardiovascular events in patients with ACS following PCI did not differ significantly between premature and late-onset ACS groups. Taken together, these results suggest that female patients should be closely observed for early risk factors of premature ACS to prevent and reduce the occurrence of adverse cardiovascular events in patients with ACS following PCI.

\section{Introduction}

Cardiovascular diseases are the leading cause of death worldwide, and coronary artery disease (CAD) is the most common cardiovascular disease with a prevalence that increases with age (1). Recent studies have demonstrated that the prevalence of CAD in the young population has been increasing $(2,3)$. The increasing prevalence of CAD in aging and young populations is associated with modifiable risk factors, which include obesity and dyslipidemia as well as other contributing risk factors, which include age and sex (2). It is well recognized that CAD results from interactions among genetic, metabolic and environmental cues (4). Based on the age at disease onset, CAD can be classified into two groups: Premature CAD and late-onset CAD (defined as disease onset at $>55$ years in men and $>65$ years in women). Premature CAD is defined as disease onset at $\leq 55$ years in men and $\leq 65$ years in women (5). Clinicopathological characteristics, independent risk factors and major adverse cardiovascular events for premature and late-onset CAD have been previously studied (6-9). Previous studies have indicated that premature CAD is associated with low levels of testosterone in young men (6) and strong familial risk (7); however, another study has indicated that patients with premature CAD exhibit severely impaired fibrinolytic response to vascular occlusion (8). In addition, the risk factors for premature and late-onset CAD differ. Smoking, family history of CAD and dyslipidemia were identified as major risk factors for premature CAD, while hypertension and diabetes were identified as major risk factors for late-onset CAD (9).

Acute coronary syndrome (ACS) is a serious type of CAD associated with the rapid progression of atherosclerotic plaques, which are predisposed to rupture and are the main cause of coronary thrombosis $(10,11)$. ACS has a high 
mortality rate and the quality of life for patients with ACS is severely affected (12). Based on the clinical manifestations of the disease, there are three main types of ACS: Unstable angina (UAP), ST-segment elevation myocardial infarction (STEMI) and non-ST-segment elevation myocardial infarction (NSTEMI) $(13,14)$. Based on the age at disease onset, ACS can be divided into two groups: Premature and late-onset ACS, similarly to CAD (15). Previous studies identified risk factors associated with the development of premature and late-onset ACS, as well as predictors for major adverse cardiovascular events. However, there were some conflicting results $(16,17)$. In a previous study involving 909 patients with premature ACS, sex was identifed as an independent risk factor for adverse cardiovascular events, as the incidence of major adverse cardiovascular events were significantly higher in female patients compared with male patients (16). However, a multi-center prospective study involving 1,163 patients revealed that there was no sex difference in the occurrence of adverse cardiovascular events in patients with premature ACS (17). Few studies have been performed to directly and systemically compare the clinical manifestations between patients with premature ACS and late-onset ACS, as well as adverse cardiovascular events in patients with ACS following percutaneous coronary intervention (PCI), a common technique which is performed to reopen completely or partially blocked coronary vessels.

The aim of the current study was to examine the differences in demographic and baseline clinicopathological characteristics of patients with premature and late-onset ACS as well as the adverse cardiovascular events in patients with ACS following PCI treatment with regard of a number of factors including sex, blood lipid profiling, plasma glucose levels and atrial fibrillation (AF). The outcomes of the present study may provide valuable and novel insight into the clinical nature and risk factors associated with premature ACS, as well as predictors for prognosis of patients with ACS.

\section{Materials and methods}

Patient selection. A total of 726 patients (male, $\mathrm{n}=465$; female, $\mathrm{n}=261$; mean age, $64.1 \pm 10.4$ years) with ACS who underwent PCI at The First Affiliated Hospital of Bengbu Medical College between February 2015 and July 2016 were enrolled in the current study. According to the National Cholesterol Education Program Adult Treatment Panel III guidelines, premature CAD is defined by an initial disease onset at $\leq 55$ years in men and $\leq 65$ years in women (15). Participants were divided into two groups: Premature ACS (men, $n=110$; female, $n=113$; mean age, 52.7 \pm .6 years) and late-onset ACS (men, $n=355$; female, $\mathrm{n}=148$; mean age, $69.2 \pm 6.8$ years). All participants provided written informed consent prior to the study. Patients with one of the three forms of ACS (UAP, STEMI and NSTEMI) were included in the current study $(10,11)$. Patients were excluded from the study if they presented with any of the following: Severe liver and kidney dysfunction, a hematopoietic system disorder, cancer or an infectious disease. The experimental protocol was established according to the ethical guidelines of the Declaration of Helsinki and was approved by the Human Ethics Committee of the First Affiliated Hospital of Bengbu Medical College (approval no. BYYFY-2014KY04).
Diagnostic methods. According to the 2010 Chinese Guidelines for the Prevention and Treatment of Hypertension (18), the diagnostic criteria for hypertension is a systolic blood pressure $\geq 140 \mathrm{mmHg}$ and/or a diastolic blood pressure $\geq 90 \mathrm{mmHg}$. According to the 2010 Chinese Guidelines for the Prevention and Treatment of Type 2 Diabetes, the diagnostic criteria for diabetes includes: i) Exhibitng symptoms of diabetes (hyperglycemia, polyphagia, polyuria, weight loss, skin itching, blurred vision and other acute metabolic disorders) with a random blood glucose reading of $\geq 11.1 \mathrm{mmol} / \mathrm{l}$; ii) fasting blood glucose $\geq 7.0 \mathrm{mmol} / 1$; and iii) blood glucose $\geq 11.1 \mathrm{mmol} / 1$ at $2 \mathrm{~h}$ after glucose load.

Sample collection. The morning after admission to the hospital, fasting venous blood samples $(5 \mathrm{ml})$ were collected and stored in test tubes coated with heparin sodium anticoagulant. Blood samples were sent to the Blood Test Center of The First Affiliated Hospital of Bengbu Medical College to measure serum levels of glucose, lipids, C-reactive protein, total cholesterol, triglyceride, high-density lipoproteins, low-density lipoproteins, uric acid and creatinine. Notably, Creatinine levels were determined using the creatine oxidase method (19). Uric acid was measured using a uric acid test kit (Ningbo Ruiyuan Biotechnology Co., Ltd.) based on the previous publication (20).

Coronary angiography using the Judkins technique (21) was performed by experienced physicians at the Department of Cardiology of The First Affiliated Hospital of Bengbu Medical College (Bengbu, China). Clinical diagnosis and evaluation were performed based on the guidelines defined by the 2001 American ACC/AHA Guidelines for the Diagnosis and Treatment of Cardiovascular Disease (22).

An intracoronary drug-coated stent was implanted in patients with coronary stenosis $\geq 70 \%$ as evaluated by angiography and details of angiography and stenting were recorded, including the course of surgery, the number of vascular lesions, the degree of stenosis, the degree of calcification, the number of implanted stents, the site and the dose of heparin and contrast agent. The criteria for evaluation of stent implantation success was defined as $\leq 20 \%$ residual stenosis with thrombolysis in myocardial infarction (TIMI) 3 grade blood flow, which was in accordance with international practice criteria (22). The Gensini score (23) was used to quantify the degree of stenosis of each vascular lesion and each score was evaluated by two independent cardiologists.

Adverse cardiovascular events, including cardiac death, non-fatal myocardial infarction (MI), heart failure, recurrent angina, stent thrombosis, stroke, major bleeding, stent restenosis and AF were examined in each patient. Furthermore, these events were observed during hospitalization and following discharge. Follow-ups were conducted by telephone or monthly outpatient visits for an average of $23.5 \pm 5.3$ months.

Routine perioperative treatments included, the administration of aspirin, clopidogrel/ticagrelor, low molecular weight heparin, statins, isosorbide mononitrate tablets, angiotensin converting enzyme inhibitors/angiotensin II receptor blocker or metoprolol sustained-release tablets, depending on the heart rate and blood pressure of the individual patient. 
Table I. Comparison of demographic and baseline clinicopathological characteristics of patients with premature and late-onset ACS.

\begin{tabular}{|c|c|c|c|c|}
\hline Characteristics & $\begin{array}{l}\text { Premature ACS } \\
\qquad(n=223)\end{array}$ & $\begin{array}{l}\text { Late-onset ACS } \\
\qquad(\mathrm{n}=503)\end{array}$ & $\chi^{2}$ & P-value \\
\hline Average age, years & $52.7 \pm 7.6$ & $69.2 \pm 6.8$ & & $<0.001$ \\
\hline Female, n $(\%)$ & $113(51)$ & $148(29)$ & 30.297 & $<0.001$ \\
\hline Male, n (\%) & $110(49)$ & $355(71)$ & 30.297 & $<0.001$ \\
\hline Smoking, n (\%) & $38(17)$ & 98 (19) & 0.606 & 0.436 \\
\hline Hypertension, n (\%) & $113(51)$ & $300(60)$ & 5.068 & 0.024 \\
\hline AMI, n (\%) & $73(33)$ & $149(30)$ & 0.705 & 0.401 \\
\hline UAP, n (\%) & $150(67)$ & $354(70)$ & 0.705 & 0.401 \\
\hline Diabetes, n (\%) & $66(30)$ & $118(23)$ & 3.076 & 0.079 \\
\hline $\mathrm{AF}, \mathrm{n}(\%)$ & $2(0.9)$ & $22(4.4)$ & 5.843 & 0.016 \\
\hline \multicolumn{5}{|l|}{ Biochemical indexes } \\
\hline $\mathrm{G}(\mathrm{mmol} / \mathrm{l})$ & $6.22 \pm 2.71$ & $5.76 \pm 2.64$ & & 0.016 \\
\hline $\mathrm{TC}(\mathrm{mmol} / \mathrm{l})$ & $4.67 \pm 1.20$ & $4.33 \pm 1.09$ & & $<0.001$ \\
\hline TG (mmol/l) & $2.05 \pm 1.68$ & $1.70 \pm 1.21$ & & $<0.001$ \\
\hline $\operatorname{HDL}(\mathrm{mmol} / \mathrm{l})$ & $1.05 \pm 0.57$ & $1.00 \pm 0.28$ & & 0.761 \\
\hline $\mathrm{LDL}(\mathrm{mmol} / \mathrm{l})$ & $2.62 \pm 0.95$ & $2.45 \pm 0.89$ & & 0.015 \\
\hline $\mathrm{CRP}(\mathrm{mg} / \mathrm{l})$ & $9.45 \pm 24.47$ & $8.23 \pm 18.38$ & & 0.464 \\
\hline $\mathrm{UA}(\mu \mathrm{mol} / \mathrm{l})$ & $308.51 \pm 83.30$ & $327.26 \pm 98.78$ & & $<0.001$ \\
\hline $\mathrm{CR}(\mu \mathrm{mol} / \mathrm{l})$ & $72.40 \pm 14.96$ & $80.69 \pm 20.97$ & & $<0.001$ \\
\hline \multicolumn{5}{|l|}{ Treatment, n (\%) } \\
\hline Bayaspirin & $223(100)$ & $503(100)$ & - & - \\
\hline Clopidogrel & $176(79)$ & $415(83)$ & 1.309 & 0.253 \\
\hline Ticagrelor & $47(21)$ & $88(17)$ & 1.309 & 0.253 \\
\hline Isosorbide mononitrate & $161(72)$ & $392(78)$ & 2.800 & 0.094 \\
\hline Statins & $223(100)$ & $503(100)$ & - & - \\
\hline LMW heparin & $223(100)$ & $503(100)$ & - & - \\
\hline ACEI/ARB & $183(82)$ & $428(85)$ & 1.062 & 0.303 \\
\hline Metoprolol SRT & $172(77)$ & $417(83)$ & 3.363 & 0.067 \\
\hline Glucose lowering agents & $52(79)$ & $105(89)$ & 3.514 & 0.061 \\
\hline
\end{tabular}

ACS, acute coronary syndrome; AMI, acute myocardial infarction; UAP, unstable angina; AF, atrial fibrillation; G, fasting plasma glucose levels; TC, total cholesterol; TG, triglyceride; HDL, high-density lipoproteins; LDL, low-density lipoproteins; CRP, C-reactive protein; UA, uric acid; CR, creatinine; LMW, low molecular weight; ACEI, angiotensin converting enzyme inhibitors; ARB, angiotensin II receptor blocker; SRT, sustained release tablets.

Statistical analysis. Data are expressed as the mean \pm standard deviation. All statistical analyses were performed using SPSS software (version 21.0; IBM Corp., Armonk, NY,USA). Normal distribution data were analyzed using a two-tailed independent t-test, and non-normally distributed data were analyzed using a Mann-Whitney U test. Classification data were presented as composition ratios and compared using the chi-squared test. The Fisher exact test was used if the theoretical number $\mathrm{T}<1$ or $\mathrm{n}<40$. Multiple linear regression analysis was used to examine the clinicaopathological features in pateints with ACS. $\mathrm{P}<0.05$ was considered to indicate a statistically significant difference.

\section{Results}

Comparison of demographic and baseline clinicopathological characteristics in patients with premature and late-onset
$A C S$. A comparison of the demographic and baseline clinicopathological characteristics between the two ACS groups is summarised in Table I. There were no significant differences with regard to smoking, diabetes mellitus, serum levels of high-density lipoprotein and C-reactive protein, baseline treatment of CAD and ACS composition ratio between the two groups $(\mathrm{P}>0.05)$. As expected, patients with premature ACS were younger than the patients with late-onset ACS. The number of female patients in the premature ACS group was significantly higher compared with that in the late-onset ACS group $(\mathrm{P}<0.001$; Table I). In addition, the serum levels of fasting blood glucose, total cholesterol, triglycerides (TG) and low-density lipoprotein (LDL) were also significantly higher in the premature ACS group compared with the late-onset ACS group $(\mathrm{P}<0.05$; Table I). Furthermore, the levels of uric acid and creatinine as well as the incidence of AF and hypertension 
Table II. Comparison of the number of coronary artery lesions and Gensini scores in patients with premature and late-onset ACS.

\begin{tabular}{lcrr}
\hline Variables & Premature ACS (n=223) & Late-onset ACS (n=503) & $\chi^{2}$ \\
\hline Three vascular lesions, n (\%) & $83(37)$ & $251(50)$ & 10.002 \\
Two vascular lesions, n (\%) & $48(22)$ & $139(28)$ & 0.002 \\
One vascular lesion, n (\%) & $92(41)$ & $113(22)$ & 0.082 \\
Gensini score & $32.44 \pm 26.85$ & $41.51 \pm 32.21$ & 26.921 \\
\hline
\end{tabular}

ACS, acute coronary syndrome.

Table III. Adverse cardiovascular events in patients with premature and late-onset ACS during hospitalization and in the follow-up period.

\begin{tabular}{lcrr}
\hline Adverse cardiovascular events & Premature ACS $(\mathrm{n}=223)$ & Late-onset ACS $(\mathrm{n}=503)$ & $\chi^{2} /$ Fisher \\
\hline Cardiac death & 0 & 11 & 0.022 \\
Non-fatal MI & 0 & 2 & $>0.05$ \\
Heart failure & 3 & 18 & 0.098 \\
Recurrent angina & 12 & 14 & 0.082 \\
Stent thrombosis & 1 & 1 & 0.520 \\
Stroke & 2 & 3 & 0.654 \\
Major bleeding & 1 & 1 & 0.520 \\
Stent restenosis & 9 & 24 & 0.020 \\
Total cardiovascular events & 28 & 74 & 0.193 \\
\hline
\end{tabular}

ACS, acute coronary syndrome; MI, myocardial infarction.

were significantly lower in the premature ACS group compared with the late-onset ACS group. Taken together, these results indicate that a sex difference exists between patients in the premature and the late-onset ACS groups and that patients with premature ACS exhibit more severe dyslipidemia compared with patients with late-onset ACS.

Comparison of coronary artery lesions and Gensini scores in patients with premature and late-onset ACS. A comparison of the number of diseased vessels and the Gensini score between the two ACS groups is summarised in Table II. The incidence of $\geq 2$ vascular lesions as well as the Gensini score in the premature ACS group was lower, but the incidence of a single vascular lesion was higher compared with that in the late-onset ACS group according to the percentages indicated (Table II). These results indicate that patients with premature ACS may have better circulation and less severe vascular lesions compared with patients with late-onset ACS.

Comparison of the adverse cardiovascular events in patients with premature and late-onset ACS during hospitalization and follow-up period. The occurrence of adverse cardiovascular events between the two ACS groups following PCI is summarized in Table III. The adverse cardiovascular events included cardiac death, non-fatal MI, heart failure, recurrent angina, stent thrombosis, stroke, major bleeding, stent restenosis and AF. All patients were closely observed during hospitalization and following discharge. Follow-ups were conducted by phone interview or monthly outpatient visits for an average of 23.5 \pm 5.3 months. There were no significant differences in the rate of occurrence of most adverse cardiovascular events between the two groups. However, the incidence of cardiac death was significantly lower in patients with premature ACS compared with patients with late-onset ACS $(\mathrm{P}<0.05$; Table III).

Multivariate logistic regression analysis offactors influencing $A C S$. Multivariate logistic regression analysis was performed to determine the factors, which may influence the pathogenesis of ACS (Table IV). The results demonstrated that sex and hypertriglyceridemia were independent risk factors, whereas low blood pressure and low incidence of AF were protective factors for premature ACS. Furthermore, multivariate logistic regression analysis was performed to determine factors, which may influence the risk of cardiovascular events in patients with ACS following PCI (Table V). The results demonstrated that age, hypertension and a high Gensini score were independent risk factors for adverse cardiovascular events in patients with ACS following PCI.

\section{Discussion}

Although the majority of people with ACS are elderly, the proportion of young people with ACS is increasing. This is mainly due to increasing rates of obesity and diabetes, as well as unhealthy lifestyles, which include smoking and a lack 
Table IV. Multivariate logistic regression analysis of the clinicopathological and familial characteristics of patients with premature ACS.

\begin{tabular}{|c|c|c|c|c|c|}
\hline Characteristics & $\beta$ & SEM & Wald & OR $(95 \% \mathrm{CI})$ & P-value \\
\hline $\begin{array}{l}\text { Sex } \\
\text { Female } \\
\text { Male }\end{array}$ & -0.935 & 0.185 & 25.644 & $0.392(0.273-0.564)$ & $<0.001$ \\
\hline $\mathrm{TC}(\mathrm{mmol} / \mathrm{l})$ & -0.103 & 0.150 & 0.466 & $0.903(0.673-1.211)$ & 0.495 \\
\hline $\mathrm{TG}(\mathrm{mmol} / \mathrm{l})$ & -0.151 & 0.075 & 4.028 & $0.860(0.742-0.996)$ & 0.045 \\
\hline LDL (mmol/l) & -0.016 & 0.175 & 0.008 & $0.984(0.699-1.387)$ & 0.929 \\
\hline $\mathrm{G}(\mathrm{mmol} / \mathrm{l})$ & -0.013 & 0.038 & 0.114 & $0.987(0.917-1.063)$ & 0.735 \\
\hline $\begin{array}{c}\text { HBP } \\
\text { Yes } \\
\text { No }\end{array}$ & -0.427 & 0.173 & 6.114 & $0.652(0.465-0.915)$ & 0.013 \\
\hline $\begin{array}{l}\text { Smoking } \\
\text { Yes } \\
\text { No }\end{array}$ & -0.081 & -0.081 & 0.120 & $0.922(0.584-1.457)$ & 0.729 \\
\hline $\begin{array}{l}\text { Diabetes } \\
\text { Yes } \\
\text { No }\end{array}$ & -0.234 & 0.225 & 1.080 & $0.791(0.509-1.231)$ & 0.299 \\
\hline $\begin{array}{l}\text { AF } \\
\text { Yes } \\
\text { No }\end{array}$ & -1.571 & 0.757 & 4.325 & $0.208(0.047-0.913)$ & 0.038 \\
\hline $\begin{array}{c}\text { AMI } \\
\text { Yes } \\
\text { No }\end{array}$ & -0.212 & 0.193 & 1.208 & $0.809(0.554-1.181)$ & 0.272 \\
\hline
\end{tabular}

ACS, acute coronary syndrome; $\beta$, regression coefficient; OR, odds ratio; CI, confidence interval; TC, total cholesterol; TG, triglyceride; LDL, low-density lipoproteins; G, fasting plasma glucose levels; HBP, high blood pressure; AF, atrial fibrillation; AMI, acute myocardial infarction; SEM, standard error of the mean.

of physical exercise in young people and children. With the transformation of 'biological-psychological-social' medical models and the development of physical and mental health, psychological factors can also serve an important role in the pathogenesis of ACS. Premature coronary heart disease is defined as initial disease onset at $\leq 55$ years in men and $\leq 65$ years in women. Cardiovascular risk factors, which include smoking, dyslipidemia, obesity and depression are important in the development of atherosclerotic vascular injury $(2,15)$. It is therefore important to investigate the association between cardiovascular risk factors and the pathogenesis of premature ACS (24).

In the present study, the demographic and baseline clinicopathological characteristics between patients with premature ACS and late-onset ACS were examined and revealed several major findings: i) Sex and hypertriglyceridemia were risk factors for premature ACS; ii) while patients with premature ACS appeared to exhibit more severe dyslipidemia, with less severe vessel disease and lower Gensini scores than patients with late-onset ACS; iii) low blood pressure and a low incidence of AF were protective factors for patients with premature ACS; and iv) patients with late-onset ACS had a higher incidence of cardiac death, which may be associated with the age of the patients in this group. The current study also demonstrated that patients with premature ACS had higher levels of fasting blood glucose compared with patients with late-onset ACS. However, there was no significant differences in the prevalence of diabetes between these two groups, which sugests that patients with premature ACS exhibit poor glycemic control.

Sex differences in the pathogenesis and prognosis of CAD is generally accepted. For example, men under the age of 40 years, have a higher risk than women for developing CAD (25), while the incidence of CAD in postmenopausal women is significantly greater than that in younger women (26). Thus, in the aging population, the incidence of CAD in men is comparable with that in women. In the present study, among female patiens, the incidence of premature ACS was greater than late-onset ACS. Consistent with this result, the multivariate logistic regression analysis also demonstrated that being female was an independent risk factor for premature ACS, indicating that the risk of developing premature ACS in women may be increasing. Hochner-Celnikier et al (27) studied 449 cases of patients with CAD, aged 45-65 years and reported that female patients with CAD had a higher incidence of risk factors including, hypertension, diabetes, obesity and hypercholesterolemia compared with male patients. The 2017 Chinese Experts Consensus on Prevention of Cardiovascular Disease (28) revealed that the 
Table V. Multivariate logistic regression analysis of the clinicopathological characteristics and cardiovascular events in patients with ACS following PCI.

\begin{tabular}{|c|c|c|c|c|c|}
\hline Characteristics & $\beta$ & SEM & Wald & OR $(95 \% \mathrm{CI})$ & P-value \\
\hline $\begin{array}{l}\text { Gender } \\
\text { Female } \\
\text { Male }\end{array}$ & 0.227 & 0.466 & 0.238 & $1.255(0.504-3.126)$ & 0.626 \\
\hline Age, years & -0.074 & 0.030 & 5.904 & $0.929(0.876-0.986)$ & 0.015 \\
\hline $\begin{array}{c}\text { ACS group } \\
\text { Premature } \\
\text { Late-onset }\end{array}$ & 0.510 & 0.724 & 0.497 & $1.666(0.403-6.890)$ & 0.481 \\
\hline $\begin{array}{c}\text { HBP } \\
\text { Yes } \\
\text { No }\end{array}$ & -0.867 & 0.420 & 4.257 & $0.420(0.184-0.958)$ & 0.039 \\
\hline $\begin{array}{l}\text { Smoking } \\
\text { Yes } \\
\text { No }\end{array}$ & -0.020 & 0.572 & 0.001 & $0.981(0.319-3.012)$ & 0.973 \\
\hline $\begin{array}{l}\text { Diabetes } \\
\text { Yes } \\
\text { No }\end{array}$ & -0.051 & 0.499 & 0.010 & $0.950(0.357-2.527)$ & 0.919 \\
\hline $\begin{array}{l}\mathrm{AF} \\
\text { Yes } \\
\text { No }\end{array}$ & -1.302 & 1.248 & 1.088 & $0.272(0.024-3.139)$ & 0.297 \\
\hline $\begin{array}{c}\text { AMI } \\
\text { Yes } \\
\text { No }\end{array}$ & -0.837 & 0.499 & 2.815 & $0.433(0.163-1.151)$ & 0.093 \\
\hline $\mathrm{G}(\mathrm{mmol} / \mathrm{l})$ & -0.083 & 0.074 & 1.263 & $0.920(0.795-1.064)$ & 0.261 \\
\hline $\mathrm{UA}(\mu \mathrm{mol} / \mathrm{l})$ & -0.001 & 0.002 & 0.322 & $0.999(0.994-1.003)$ & 0.570 \\
\hline $\mathrm{CR}(\mu \mathrm{mol} / \mathrm{l})$ & -0.001 & 0.011 & 0.012 & $0.999(0.978-1.020)$ & 0.914 \\
\hline $\mathrm{TC}(\mathrm{mmol} / \mathrm{l})$ & -0.053 & 0.511 & 0.011 & $0.948(0.348-2.584)$ & 0.918 \\
\hline $\mathrm{TG}(\mathrm{mmol} / \mathrm{l})$ & 0.072 & 0.266 & 0.073 & $1.075(0.637-1.812)$ & 0.787 \\
\hline $\mathrm{LDL}(\mathrm{mmol} / \mathrm{l})$ & 0.236 & 0.558 & 0.179 & $1.266(0.424-3.783)$ & 0.672 \\
\hline HDL (mmol/l) & 1.448 & 1.024 & 1.999 & $4.255(0.572-31.683)$ & 0.157 \\
\hline $\mathrm{CRP}(\mathrm{mg} / \mathrm{l})$ & 0.003 & 0.010 & 0.106 & $1.003(0.984-1.023)$ & 0.745 \\
\hline Gensini score & -0.018 & 0.008 & 5.332 & $0.982(0.968-0.997)$ & 0.021 \\
\hline Diseased vessels & -0.192 & 0.269 & 0.511 & $0.825(0.487-1.398)$ & 0.475 \\
\hline
\end{tabular}

ACS, acute coronary syndrome; PCI, percutaneous coronary intervention; $\beta$, regression coefficient; OR, odds ratio; CI, confidence interval; HBP, high blood pressure; AF, atrial fibrillation; AMI, acute myocardial infarction; G, fasting plasma glucose levels; UA, uric acid; CR, creatine; TC, total cholesterol; TG, triglyceride; LDL, low-density lipoproteins; HDL, high-density lipoprotein; CRP, C-reactive protein; SEM, standard error of the mean.

prevalence of hypertension, diabetes and hypercholesterolemia in women are increasing, which corresponds with the increasing prevalence of CAD as well as morbidity and mortality rates. Therefore, to prevent or reduce the incidence of premature CAD, blood pressure, blood glucose and lipid abnormalities need to be monitored in each sex to provide early intervention and treatments to prevent ACS.

The serum level of TG is a known independent risk factor for CAD (29). Mechanistically, elevated serum levels of TG result in the increased association of lipoprotein (a) with TG-rich lipoproteins (30), through which lipoprotein (a) selectively increases mRNA levels of a tissue-type plasminogen activator inhibitor in vascular endothelial tissues, which subsequently causes the synthesis and release of tissue-type plasminogen activator inhibitors (31). Increased activity of the plasminogen activator inhibitor is a predictor of CAD, in particular of premature CAD (32). In addition, increased plasma TG can raise the concentration of LDL (33). Previous studies have demonstrated that the cholesterol present in atherosclerotic plaques comes mainly from LDL, which has the higest percentage of cholesterol of all the lipoproteins and is the most potent lipoprotein at promoting artherosclerosis (34). In the present study, serum levels of total cholesterol, TG and LDL in patients with premature ACS were significantly higher 
comapred with patients with late-onset ACS, and multivariate logistic regression also demonstrated that a high serum level of TG was an independent risk factor for premature ACS. The results of the current study suggest that patients with premature ACS may have more severe lipid disorders. Therefore, screening and controlling blood lipid levels in the younger population may be an effective way to prevent/reduce the incidence of premature ACS.

In the present study, a comparison of the number of diseased vessels and the Gensini scores between premature and late-onset ACS groups revealed that the premature ACS group had significantly lower Gensini scores and a lower incidence of triple vascular lesions, however, the incidence of single vascular lesions was higher compared with than in the late-onset ACS group according to the percentages indicated. As the patients in the late-onset ACS group were older, these results indicate that the increased Gensini score and high incidence of triple vascular lesions may be associated with aging. Therefore, the prevention and treatment of premature ACS should be performed in order to prevent or reverse the progression of coronary lesions. In addition, the Syntax score is a method for assessing the extent of coronary lesions (35), which is based on coronary angiography results. This will be calculated and analyzed in future experiments.

While adverse cardiovascular events have been widely described for patients with late-onset ACS following PCI, few studies have examined adverse cardiovascular events in patients with premature ACS. In the present study, adverse cardiovascular events in patients with premature and late-onset ACS following PCI was examined during hospitalization and the follow-up period of $23.5 \pm 5.3$ months. There were no significance differences in the incidence of heart failure, non-fatal MI, recurrent angina, stroke, hemorrhage, in-stent restenosis and thrombosis between the premature and late-onset ACS groups. There also was no significant difference in the total incidence of adverse cardiovascular events between the premature and late-onset ACS groups. These results suggest that patients with premature or late-onset ACS have comparable risks for developing adverse cardiovascular events following PCI. By contrast, the incidence rates of $\mathrm{AF}$, hypertension and cardiac death in patients with premature ACS were significantly lower compared with patients with late-onset ACS. However, this may be attributed to the fact that patients in the late-onset ACS group were older and the incidence of AF (36), hypertension (37) and cardiac death increases with age. As patients with premature ACS are usually younger and middle-aged workers, their socioeconomic losses are expected to be much greater than those with late-onset ACS, methods for improved screening and early prevention and treatment of patients with premature ACS may reduce the potential financial burden for patients' families and society.

Previous studies revealed a link between impaired fasting plasma glucose levels and the risk of CAD with poor prognosis $(38,39)$. In addition, the present study demonstrated that fasting plasma glucose levels in patients with premature ACS were significantly higher than those in patients with late-onset ACS. However, there was no significant difference in the prevalence of diabetes between these two groups. Furthermore, while fasting plasma glucose levels were significantly higher in patients with premature ACS, there was no evidence to suggest a poorer prognosis in patients with premature ACS compared with patients with late-onset ACS. These observations indicate poor control of blood glucose levels in patients with premature ACS and given that higher fasting plasma glucose levels were identified as a risk factor for the development of ACS (40), blood gluose levels should be monitored more closely.

There are several limitations associated with the current study which should be noted. As a retrospective study, the current study has inherent limitations associated with the nature of retrospective studies, which includes potential sampling bias. In addition, the current study was performed in a single center with a relatively small sample size. Therefore, future studies with larger, multi-center cohorts should be performed in order to validate the results of the current study.

In conclusion, the present study examiend and compared the clinicopathological characteristics of patients with premature ACS and the adverse cardiovascular events in patients with ACS following PCI. Sex and hyperlipidemia were identified as risk factors for the development of premature ACS. In addition, the incidence of adverse cardiovascular events in patients with premature ACS was comparable to that of patients with late-onset ACS following PCI, despite the fact that patients with premature ACS had less severe vascular disease and patients with late-onset ACS were older. Taken together, these results suggest that early screening of high-risk patients should be performed, particularly in the female population, and plasma levels of lipids and fasting glucose should be monitored in order to prevent and reduce the occurrence of premature ACS. In addition, patients with premature ACS should be treated as early as possible to prevent the progression of ACS.

\section{Acknowledgements}

Not applicable.

\section{Funding}

The present study was supported by grants from the Science and Technology Project of Anhui Province (grant no. 1501041154), Major Projects of Natural Science Research in Universities of AnhuiProvince(grant no.KJ2015ZD30), MajorProject of Natural Science in Bengbu Medical College (grant no. BYKY1730ZD) and the Science and Technology Development Fund in Bengbu Medical College (grant no. BYKF1757).

\section{Availability of data and materials}

The datasets used and/or analyzed during the present study are available from the corresponding author on reasonable request.

\section{Authors' contributions}

XJS and MNL designed the study, conducted all literature searches, performed critical appraisal of potential studies and prepared the manuscript. LX and HL participated in data collection and follow-up. BC and JZ analyzed the data and assisted with drafting and revising the manuscript. HJW conceived and designed the study, assisted with searches, appraised relevant studies and assisted with drafting and revising the manuscript. All authors read and approved the final manuscript. 


\section{Ethics approval and consent to participate}

The experimental protocol was established according to the ethical guidelines of the Declaration of Helsinki and approved by the Human Ethics Committee of the First Affiliated Hospital of Bengbu Medical College (Bengbu, China).

\section{Patient consent for publication}

Not applicable.

\section{Competing interests}

The authors declare that they have no competing interests.

\section{References}

1. Odden MC, Coxson PG, Moran A, Lightwood JM, Goldman L and Bibbins-Domingo K: The impact of the aging population on coronary heart disease in the United States. Am J Med 124: 827-833.e5, 2011.

2. Yang L, Li L, Lewington S, Guo Y, Sherliker P, Bian Z, Collins R, Peto R, Liu Y, Yang R, et al: Outdoor temperature, blood pressure, and cardiovascular disease mortality among 23000 individuals with diagnosed cardiovascular diseases from China. Eur Heart J 36: 1178-1185, 2015.

3. Benjamin EJ, Blaha MJ, Chiuve SE, Cushman M, Das SR, Deo R, de Ferranti SD, Floyd J, Fornage M, Gillespie C, et al: Heart disease and stroke statistics-2017 Update: A report from the American heart association. Circulation 135: e146-e603, 2017.

4. Basak T, Varshney S, Hamid Z, Ghosh S, Seth S and Sengupta S: Identification of metabolic markers in coronary artery disease using an untargeted LC-MS based metabolomic approach. J Proteomics 127: 169-177, 2015.

5. Wood D, De Backer G, Faergeman O, Graham I, Mancia G and Pyörälä K: Prevention of coronary heart disease in clinical practice: Recommendations of the Second Joint Task Force of European and other Societies on Coronary Prevention. Eur Heart J 19: 1434-1503, 1998.

6. Alkamel A, Shafiee A, Jalali A, Boroumand M and Nozari Y: The association between premature coronary artery disease and level of testosterone in young adult males. Arch Iran Med 17: 545-550, 2014.

7. Scheuner MT, Whitworth WC, McGruder H, Yoon PW and Khoury MJ: Familial risk assessment for early-onset coronary heart disease. Genet Med 8: 525-531, 2006.

8. Speidl WS, Nikfardjam M, Niessner A, Jordanova N, Zorn G, Maurer G, Schreiber W, Wojta J and Huber K: Premature compared with late onset of coronary artery disease: Young patients show a severe defect in fibrinolytic response to venous occlusion. Blood Coagul Fibrinolysis 18: 165-171, 2007.

9. Reibis R, Treszl A, Wegscheider K, Bestehorn K, Karmann B and Völler H: Disparity in risk factor pattern in premature versus late-onset coronary artery disease: A survey of 15,381 patients Vasc Health Risk Manag 8: 473-481, 2012.

10. Chinese Society of Cardiology of Chinese Medical Association; Editorial Board of Chinese Journal of Cardiology: Guideline and consensus for the management of patients with non-ST-elevation acute coronary syndrome (2016). Zhonghua Xin Xue Guan Bing Za Zhi 45: 359-376, 2017.

11. Badimon L, Bugiardini R and Cubedo J: Pathophysiology of acute coronary syndromes in the elderly. Int J Cardiol 222: 1105-1109, 2016.

12. Editorial Board of Chinese Journal of Cardiologyvascular Diseases: Guidelines for the diagnosis and treatment of non-ST-segment elevation acute coronary syndrome, 2016. Chin Soc Cardiol 45: 359-376, 2017 (In Chinese).

13. Amsterdam EA, Wenger NK, Brindis RG, Casey DE Jr, Ganiats TG, Holmes DR Jr, Jaffe AS, Jneid H, Kelly RF, Kontos MC, et al: 2014 AHA/ACC guideline for the management of patients with non-ST-elevation acute coronary syndromes: A report of the American College of Cardiology/American Heart Association Task Force on Practice Guidelines. J Am Coll Cardiol 64: e139-e228, 2014
14. Ibanez B, James S, Agewall S, Antunes MJ, Bucciarelli-Ducci C, Bueno $\mathrm{H}$, Caforio ALP, Crea F, Goudevenos JA, Halvorsen S, et al: 2017 ESC Guidelines for the management of acute myocardial infarction in patients presenting with ST-segment elevation: The Task Force for the management of acute myocardial infarction in patients presenting with ST-segment elevation of the European Society of Cardiology (ESC). Eur Heart J 39: 119-177, 2018.

15. Grundy SM, Cleeman JI, Merz CN, Brewer HB Jr, Clark LT, Hunninghake DB, Pasternak RC, Smith SC Jr, Stone NJ; National Heart, Lung, and Blood Institute, et al: Implications of recent clinical trials for the National Cholesterol Education Program Adult Treatment Panel III guidelines. Circulation 110: 227-239, 2004.

16. Pelletier R, Khan NA, Cox J, Daskalopoulou SS, Eisenberg MJ, Bacon SL, Lavoie KL, Daskupta K, Rabi D, Humphries KH, et al: Sex versus gender-related characteristics: Which predicts outcome after acute coronary syndrome in the young? J Am Coll Cardiol 67: 127-135, 2016.

17. Pelletier R, Choi J, Winters N, Eisenberg MJ, Bacon SL, Cox J, Daskalopoulou SS, Lavoie KL, Karp I, Shimony A, et al: Sex differences in clinical outcomes after premature acute coronary syndrome. Can J Cardiol 32: 1447-1453, 2016.

18. 2010 Chinese guidelines for the prevention and treatment of hypertension. Chin J front Med Sci (Electronic Version) 3: 42-90, 2011 (In Chinese).

19. Xu, Guobin Zhu and Lihua Xia, Tie'an: Determination of creatinine dehydrogenase by creatinine and amidine hydrolase by creatinine enzymatic method. J Clin Laboratory Med 19: 149, 2001.

20. Gochman N and Schmitz JM: Automated determination of uric acid, with use of a uricase-peroxidase system. Clin Chem 17: 1154-1159, 1971

21. Harry L and Page Jr MD: The Judkins technique. Catheter cardiovasc interv 5: 187-189, 1979.

22. Smith SC Jr, Dove JT, Jacobs AK, Kennedy JW, Kereiakes D, Kern MJ, Kuntz RE, Popma JJ, Schaff HV, Williams DO, et al: ACC/AHA guidelines for percutaneous coronary intervention (revision of the 1993 PTCA guidelines)-executive summary: A report of the American College of Cardiology/American Heart Association task force on practice guidelines (Committee to revise the 1993 guidelines for percutaneous transluminal coronary angioplasty) endorsed by the Society for Cardiac Angiography and Interventions. Circulation 103: 3019-3041, 2001.

23. Gensini GG: A more meaningful scoring system for determining the severity of coronary heart disease. Am J Cardiol 51: 606, 1983.

24. Wei W, Zhijun W and Xinying C: Early onset and late onset coronary heart disease risk factors of coronary artery disease. World Latest Med Inform (Electronic Version) Vol 16, No. 8: 18-20, 2016

25. Lloyd-Jones DM, Larson MG, Beiser A and Levy D: Lifetime risk of developing coronary heart disease. Lancet 353: 89-92, 1999.

26. Dosi R, Bhatt N, Shah P and Patell R: Cardiovascular disease and menopause. J Clin Diagn Res 8: 62-64, 2014.

27. Hochner-Celnikier D, Manor O, Gotzman O, Lotan H and Chajek-Shaul T: Gender gap in coronary artery disease: Comparison of the extent, severity and risk factors in men and women aged 45-65 years. Cardiology 97: 18-23, 2002.

28. Committee of Women Physician in Chinese College Cardiovascular Physicians; Women's Health Work Group in Chinese Society of Cardiology: Expert consensus for the prevention of cardiovascular disease in Chinese women. Zhonghua Nei Ke Za Zhi 56: 472-476, 2017 (In Chinese).

29. Park JY,Lee SH, Shin MJ and Hwang GS: Alteration in metabolic signature and lipid metabolism in patients with angina pectoris and myocardial infarction. PLoS One 10: e 0135228, 2015.

30. Chapman MJ, Ginsberg HN, Amarenco P, Andreotti F, Borén J, Catapano AL, Descamps OS, Fisher E, Kovanen PT, Kuivenhoven JA, et al: Triglyceride-rich lipoproteins and high-density lipoprotein cholesterol in patients at high risk of cardiovascular disease: Evidence and guidance for management. Eur Heart J 32: 1345-1361, 2011.

31. Kugiyama K, Sakamoto T, Misumi I, Sugiyama S, Ohgushi M, Ogawa H, Horiguchi M and Yasue H: Transferable lipids in oxidized low-density lipoprotein stimulate plasminogen activator inhibitor-1 and inhibit tissue-type plasminogen activator release from endothelial cells. Circ Res 73: 335-343, 1993. 
32. van de Woestijne AP, Wassink AM, Monajemi H, Liem AH, Nathoe HM, van der Graaf Y and Visseren FL; SMART study group: Plasma triglyceride levels increase the risk for recurrent vascular events independent of LDL-cholesterol or nonHDL-cholesterol. Int J Cardiol 167: 403-408, 2013.

33. Jellinger PS, Handelsman Y, Rosenblit PD, Bloomgarden ZT, Fonseca VA, Garber AJ, Grunberger G, Guerin CK, Bell DSH and Mechanick JI: American Association Of Clinical Endocrinologists And American College Of Endocrinology Guidelines For Management Of Dyslipidemia And Prevention Of Cardiovascular Disease. Endocr Pract 23 (Suppl 2): 1-87, 2017.

34. Jellinger PS, Handelsman Y, Rosenblit PD, Bloomgarden ZT, Fonseca VA, Garber AJ, Grunberger G, Guerin CK, Bell DSH, Mechanick JI, et al: American association of clinical endocrinologists and American college of endocrinology guidelines for management of dyslipidemia and prevention of cardiovascular disease. Endocr Pract 23 (Suppl 2): S1-S87, 2017.

35. Sianos G, Morel MA, Kappetein AP, Morice MC, Colombo A, Dawkins K, van den Brand M, Van Dyck N, Russell ME, Mohr FW and Serruys PW: The SYNTAX score: An angiographic tool grading the complexity of coronary artery disease. EuroIntervention 1:219-227, 2005.

36. Mairesse GH, Moran P, Van Gelder IC, Elsner C, Rosenqvist M, Mant J, Banerjee A, Gorenek B, Brachmann J, Varma N, et al: Screening for atrial fibrillation: A European Heart Rhythm Association (EHRA) consensus document endorsed by the Heart Rhythm Society (HRS), Asia Pacific Heart Rhythm Society (APHRS), and Sociedad Latinoamericana de Estimulación Cardíaca y Electrofisiología (SOLAECE). Europace 19: 1589-1623, 2017.
37. Li R: Guide to Prevention and Treatment of Essential Hypertension in China 2010: Introduction 2010 Essentials. Chin J General Pract 11: 8-10, 2012 (In Chinese).

38. Shahim B, De Bacquer D, De Backer G, Gyberg V, Kotseva K, Mellbin L, Schnell O, Tuomilehto J, Wood D, Rydén L, et al: The prognostic value of fasting plasma glucose, two-hour postload glucose, and $\mathrm{HbA}_{\mathrm{c}}$ in patients with coronary artery disease: A report from EUROASPIRE IV: A survey from the european society of cardiology. Diabetes Care 40: 1233-1240, 2017.

39. Emerging Risk Factors Collaboration, Sarwar N, Gao P, Seshasai SR, Gobin R, Kaptoge S, Di Angelantonio E, Ingelsson E, Lawlor DA, Selvin E, et al: Diabetes mellitus, fasting blood glucose concentration, and risk of vascular disease: A collaborative meta-analysis of 102 prospective studies. Lancet 375: 2215-2222, 2010.

40. Zhang X, Dong L, Wang Q and Xie X: The relationship between fasting plasma glucose and MPO in patients with acute coronary syndrome. BMC Cardiovasc Disord 15: 93, 2015. 